\title{
NEUTRAL HYDROGEN IN THE CENTRAL PART OF THE GALACTIC SYSTEM
}

\author{
G. W. ROUGOOR AND J. H. OORT \\ Leiden Observatory, The Netherlands
}

\section{INTRODUCTION}

While trying to determine the rotation curve in $1953 \mathrm{Kwee}$, Muller, and Westerhout [1] found long and faint wings in the line profiles within 20 to 25 degrees longitude from the center. The gas responsible for these wings should therefore lie within 3 kiloparsecs from the center. The velocities of the neutral hydrogen causing these wings are far greater than could be expected on the basis of a reasonable rotational model of the Galaxy. Therefore, the wings were tentatively interpreted as being caused by high turbulent velocities in the gas. In view of the new and better data obtained with the 25-meter telescope in Dwingeloo, this interpretation has now been dropped. The new conclusion is that all of the neutral hydrogen in these regions is expanding and at the same time taking part in the galactic rotation. The evidence for this conclusion will be briefly presented.

The phenomenon occurs in a very narrow range of latitudes, mainly between $b=-0.5$ and $b=-2.5$. In Fig. 1 some features of the line profiles in the region in which we are interested are plotted as a function of galactic

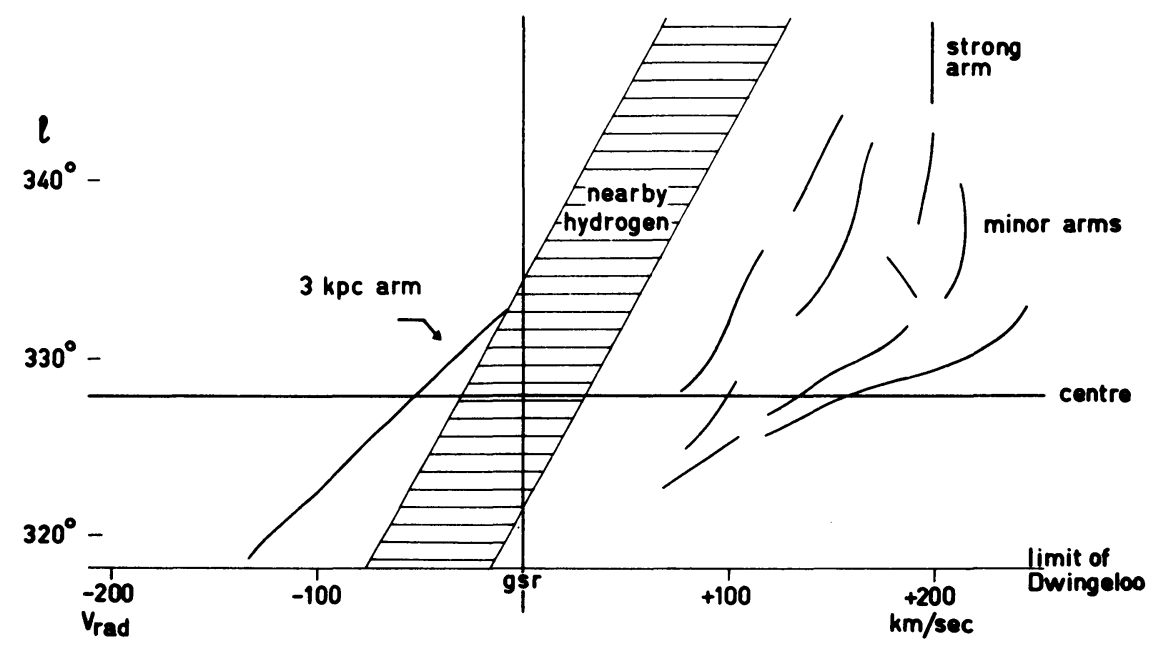

FIG. 1. Location of studied features in a longitude-velocity diagram. The velocities are relative to the galactic center of rest, computed with $v_{\odot}=216 \mathrm{~km} / \mathrm{second}$. 


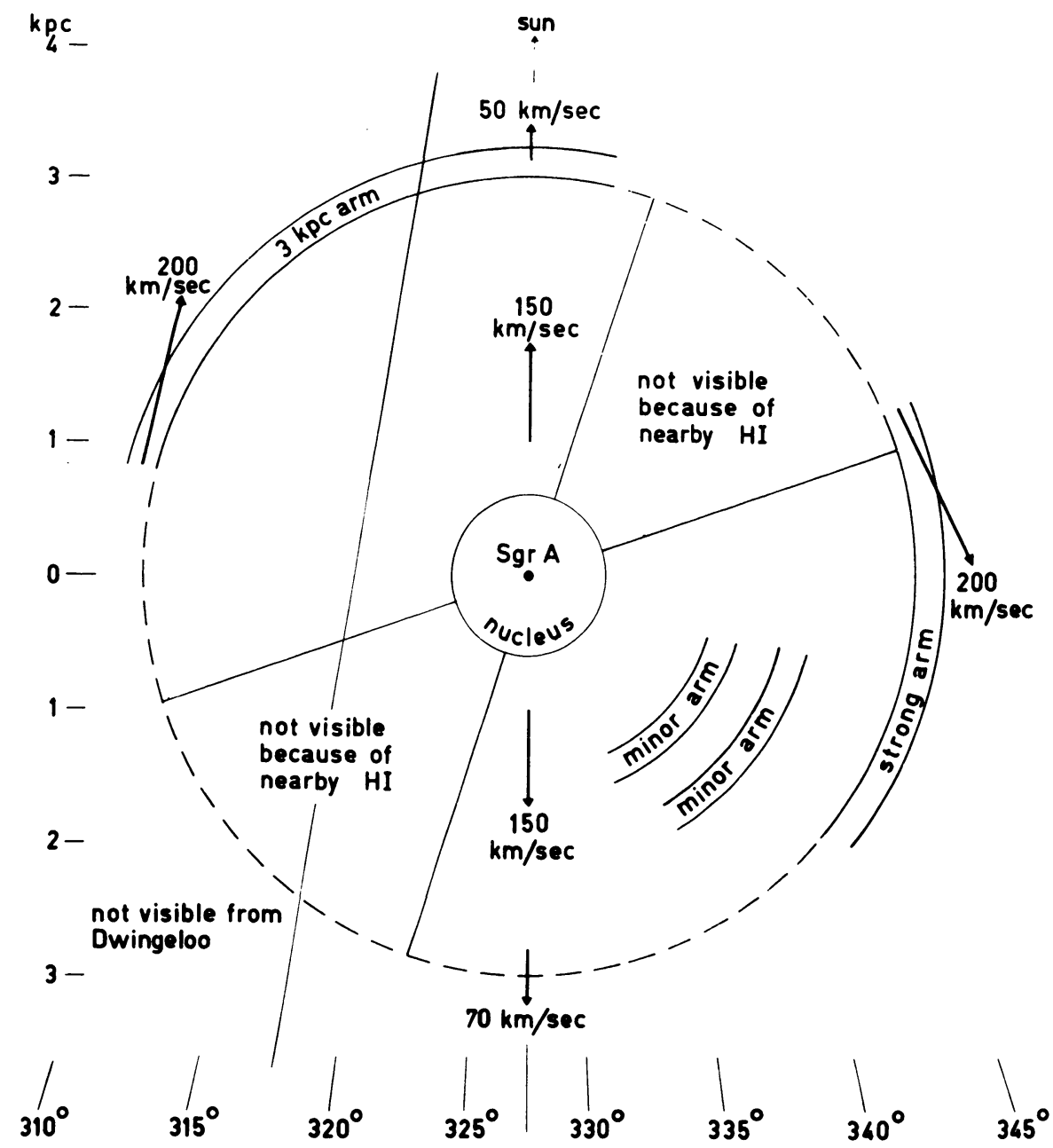

FIG. 2. Map of the central region of the galactic system showing probable location of several arms.

longitude and radial velocity. The map of the central region in Fig. 2 shows the probable location of these features in the Galaxy.

First we shall discuss the observations made in the direction of the galactic center. This direction is given in Fig. 1 as a horizontal line at $l=327.7$. Here the interpretation is relatively clear. Second we shall discuss the situation at other longitudes and attempt to determine the true motion and geometry in these regions.

\section{CENTER DiRECTION (SAGitTaRIUS A)}

As a working hypothesis we assume that Sagittarius A is situated in the center of the galactic system. There is now considerable evidence in support of this assumption. 


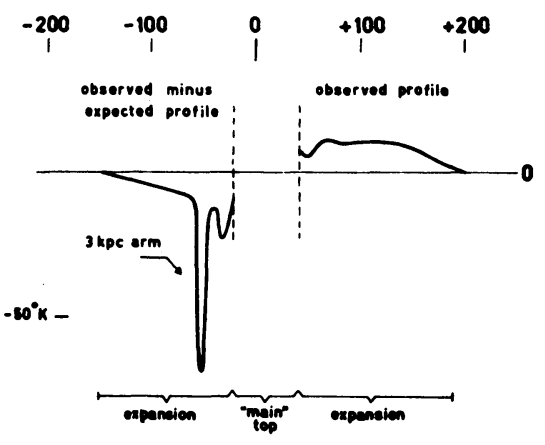

FIG. 3. Cross sections in galactic latitude through Sagittarius A.

The absorption and emission in the direction of Sagittarius A may be seen in Fig. 3. The main emission and the absorption lines with small velocity, due to nearby hydrogen, have been omitted.

The conspicuous absorption line at $-53 \mathrm{~km} / \mathrm{second}$ is a rather dense arm that passes in front of the source [2]. Hydrogen moving in circular orbits around the galactic center has a zero radial-velocity component in this direction. The negative velocity of this arm is therefore entirely the result

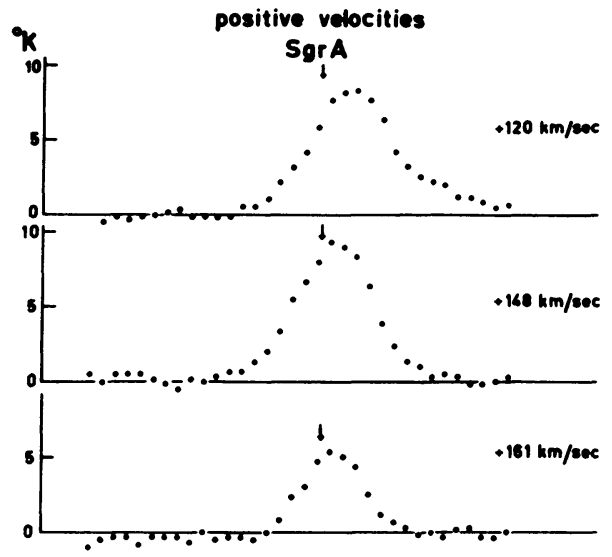

negative velocities
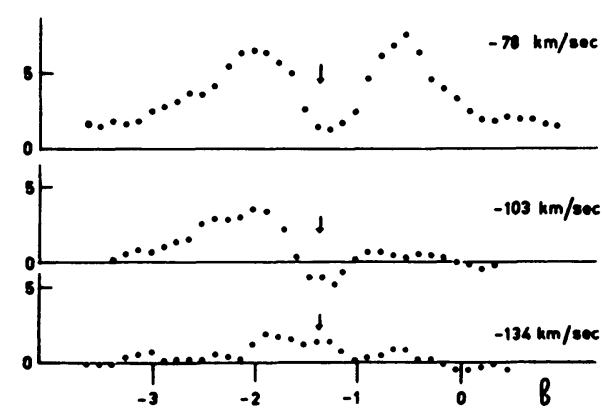

Fig. 4. Absorption and emission profile at Sagittarius A. of motion toward the sun, away from the center (expansion). The inference that the expansion is a general phenomenon in the central region is based on the fact that Fig. 3 shows absorption at all negative velocities up to $-150 \mathrm{~km} / \mathrm{second}$ (hydrogen in front of the source) and no absorption at the corresponding positive velocities (hydrogen behind the source). Fig. 3 is based on cross-sections in latitude, samples of which are shown in Fig. 4. Although no absorption dip is visible at $v=-134 \mathrm{~km} /$ second, absorption is probable if we assume a symmetry of negative and positive velocities.

The fact that the source lies at the center of the expansion lends additional support to the supposition that it is situated in the center of the Galaxy.

\section{ARMS IN THE CENTRAL REGION}

The continuous ridges in Fig. 1 probably have to be interpreted as arms, although there is no evidence at all for a spiral shape. The rather dense arm denoted as " $3-\mathrm{kpc}$ arm" is seen tangentially at $l=305$ degrees (Australian 
observations), from which its distance $R=3$ kiloparsecs is inferred [3]. The Dwingeloo observations extend from $l=318$ degrees upwards. The 3-kiloparsec arm disappears into the main peak of the line profiles (Fig. 1) at about $l=334$ degrees and therefore cannot be traced further. It has half-widths in velocity and latitude of $16 \mathrm{~km} /$ second and 1.5 , and a maximum brightness temperature of $40^{\circ} \mathrm{K}$. With $T_{0}=125^{\circ} \mathrm{K}$ this gives an optical depth $\tau=0.4$, in close agreement with the value $\tau=0.5$ found from the absorption profile. In the latter computation the temperature of the hydrogen does not have to be taken into account; we may conclude, therefore, that even at this distance from the galactic center the kinetic temperature is not significantly different from that in the solar neighborhood.

In the region $l=343$ to 334 degrees a number of ridges in the line profiles are visible, each extending over a few degrees in longitude, with intensities around $15^{\circ} \mathrm{K}$ and half-widths in velocity and latitude of $20 \mathrm{~km} / \mathrm{second}$ and 1 degree. Their velocities of recession are far too great to be explained by rotational motion only, and must therefore contain a large expansion component.

The ridges below $l=334$ degrees have a much lower intensity and a greater velocity width. At longitudes smaller than $327: 7$ their velocity is opposite to the normal rotational velocities ("forbidden velocities"). The ridge at $l=323$ degrees is very conspicuous and its shape and motion indicate that it is a rather concentrated minor arm expanding with a velocity of at least $150 \mathrm{~km} /$ second.

As neither the variation of the velocity of rotation nor that of the systematic motion away from the center are known, it is not possible to find the distance from the center for these individual bits. The indications "minor arm" in Fig. 2 have no more than schematic significance.

It may be, in fact, that the ridges with higher velocities form a brokenup extension of the "strong arm," which sets in at about 340 degrees. This arm would then be visible from $l=323$ to $l=350$ degrees and would have an expansional motion of about $130 \mathrm{~km} / \mathrm{second}$. At 350 degrees it would be about 3 kiloparsecs from the center, while on this hypothesis its distance at the lower longitudes would have to be somewhat smaller. It might form a counterpart of the 3-kiloparsec expanding arm on the other side of the center.

The profiles at negative velocities have not yet been fully investigated, with the exception of the " $3-\mathrm{kpc}$ arm" and the absorption at the position of Sagittarius A.

\section{EXPANSION MECHANISM}

At $R=3$ kiloparsecs the neutral hydrogen is apparently expanding with velocities between 50 and $130 \mathrm{~km} / \mathrm{second}$. If we take the velocity to be 50 $\mathrm{km} / \mathrm{second}$ and assume a total average density of $0.3 \mathrm{~cm}^{-3}$ at $R=3$ kiloparsecs and a layer thickness of 150 parsecs, the total amount of gas streaming through a cylinder with radius $R=3$ kiloparsecs is about $1 M_{\odot}$ per year. During the Galaxy's lifetime this would amount to $5 \times 10^{9} M_{\odot}$, or about 10 per cent of the total mass of the galactic system.

In order to balance the loss caused by this outward streaming, the central 
disk must be replenished either by condensation from the galactic corona or by gas expelled from stars in the central region. A rough calculation, based on data kindly provided by Schmidt [4], shows that the present rate of ejection by stars would be much smaller than the amount needed. Condensation from the corona into the disk seems, therefore, the only plausible way of replenishment.

The mechanism that causes the expansion of the central arms must probably be sought in a concentration of the interstellar magnetic field near the galactic nucleus.

We are greatly indebted to $\mathrm{Mr}$. Muller for the care and thought he has given to improving the stability of the receiver and to keeping it in perfect condition during these observations. Without this permanent attention successful observations of the wings of low intensity would not have been possible.

\section{REFERENCES}

[1] Kwee, K. K., Muller, C. A., and Westerhout, G. B.A.N. 12, 211, 1954.

[2] van Woerden, H., Rougoor, W., and Oort, J. C.R. 244, 1691, 1957.

[3] Kerr, F. J. Private communication.

[4] Schmidt, M. Article to be published in the $A p . J$.

\section{Discussion}

Davies: The Sagittarius A source has two parts: one large, of diameter greater than one degree; the other a point source. Are both parts at the center?

Rougoor: Most of the continuous radiation is in the center. It may be that part of the source consists of a nearby component, but that doesn't affect the expansion theory.

McVittie: If the supply of gas comes from stars of Population II, are there enough stars of this kind emitting matter to keep up the quantity needed? Has anyone made an estimate of the production of gas by stars near the galactic center?

Oort: We do not know at all whether at the present epoch Population II stars eject important amounts of gas. In any case the total amount needed would seem to be too high to come from such stars.

van de Hulst: It is not really sufficient to have a peculiar type of star that ejects matter near the center. This material would soon be drawn back by gravitation, if our ideas of the gravitational potential are correct. So a driving force of unknown origin has to keep up the motion through the entire region.

Heeschen: How does the expansion velocity vary with distance from the center?

Oort: In reply to Heeschen's question I should like to point out that the 
observations provide no answer, because we do not know the distances from the center of the features observed in this part of the Galaxy. However, if the supply for the outflowing matter comes from the galactic corona it seems probable that the radial motion would increase with increasing radius. It seems hardly possible to have all this matter streaming in very near the nucleus, if only because it could not then cool down rapidly enough. It seems much more probable that it would condense over a large part of the disk with radius of about 3 kiloparsecs. A homogeneous corona would produce an outward velocity proportional to radius. Near a radius of 3 kiloparsecs the mechanism apparently stops and the expanding velocity decreases rapidly.

Schatzman: Does anyone have an idea of the degree of hydrogen ionization in the central part of the Galaxy?

Westerhout: From the $22-\mathrm{cm}$ survey of the continuous radiation, and comparing this with Dr. Mills' 3.5-m survey, I have made an estimate of the distribution of ionized hydrogen in the Galaxy. It appears that within 3 kiloparsecs the $\mathrm{H}$ II density is at least a factor 2 to 5 smaller than in the region from 3 to 6 kiloparsecs, and that the total amount of $\mathrm{H}$ II in the central region is not more than 10 per cent of the total amount of $\mathrm{H} \mathrm{I}$, if we assume that the ionized hydrogen still exists in the form of discrete clouds. If the medium is continuous (no clouds) the $\mathrm{H}$ II density may be comparable to the $\mathrm{HI}$ density. The figures given here are upper limits. The rough model fitted to the observations gives zero $\mathrm{H}$ II density inside 2 kiloparsecs.

van de Hulst: The usual estimates of the ionization that exists even in $\mathrm{H}$ I regions give a conductivity that is amply sufficient to apply hydromagnetic reasoning to the gas in the central region.

Bracewell: Could Professor Gold comment on the compatibility of $\mathrm{Mr}$. Rougoor's observations of radially outward flow from Sagittarius A with the inward flow near the center resulting from the redistribution of matter under the influence of internal friction?

Gold: On the assumption of an angular velocity of gravitational orbits that diminishes outward, there would be a change-over point between inward and outward motion of the gas if this is subjected to some internal frictional force. The region one observes may be larger than the inner zone of this pattern, and one thus observes the outward flow.

Oort: Is it possible to reconcile the suggestions of Dr. Gold with the fact that no inward motions are observed?

Blaauw: Is the thickness of the layer within 3 kiloparsecs well determined? Could it be that, for instance, it is thicker in the nucleus?

Rougoor: The thickness of the layer is constant within 3 kiloparsecs from the center. At negative velocities (3-kiloparsec arm) the width to half-power points is about 1.5 degrees, at positive velocities the width is about 1.0 degrees. This may be a distance effect but the results are very uncertain.

Lilley: The amount of gas deduced from the measurements is critical both to the question of the origin of the gas and to the driving mechanism for 
the gas. Is the figure you give for the expanding gas taken from the expected profile or from the derived opacity at an assumed state temperature?

Rougoor: In the case of the 3-kiloparsec arm we can derive an optical depth from the observed profile at the position of Sagittarius A and from the expected profile. No assumption on kinetic temperature, therefore, is necessary. The expected profile has been estimated from scans at constant declination at all velocities which define the dip in the absorption profile. The difference gives the true absorption and hence the optical depth. The derived optical depth is about 0.5 . We can see whether this value fits the emission profiles at $l=318$ to $l=325$ degrees. The maximum antenna temperature found here is about $50^{\circ} \mathrm{K}$, which together with the optical depth found above corresponds to a kinetic temperature of about $120^{\circ} \mathrm{K}$. This value is of the same order as found elsewhere in the galactic system.

Hoyle: I would like to mention a third possible driving mechanism. The rotation curve shown by $\mathrm{Dr}$. Oort would seem to imply that over $5 \times 10^{\circ}$ years any magnetic field associated with the spiral structure of the Galaxy ought to have many more windings than in fact we find it to have. The question therefore seems to arise about how a spirally wound magnetic field can lose turns. One possibility is that the turns are pushed outside the galactic disk, out into intergalactic space. Another possibility is that the problem does not remain a purely two-dimensional one; that the turns slip over each other in the third dimension. All the indications are that this would be most likely to occur in the central regions, where the magnetic pressure is probably a maximum, where the gas density is low, and where the distances of slip are not very large. It may be that we are observing a stage of this slipping process, possibly a stage where a slipover has just taken place. 